\title{
Dehydrodiisoeugenol inhibits colorectal cancer growth by endoplasmic reticulum stress induced autophagic pathways
}

Changhong Li 1,2, 4, ${ }^{*}$, Kui Zhang ${ }^{1,4,}$, Guangzhao Pan ${ }^{1,4}$, Haoyan $\mathrm{Ji}^{1,4}$, Chongyang Li, Xiaowen Wang ${ }^{1,4}$, Yi

Wang ${ }^{5}$, Ruochen Liu ${ }^{1,4}$, Longfei Deng ${ }^{2}$, Liqun Yang 1, 4, \#, Hongjuan Cui 1,2,3, 4, 5

1. State Key Laboratory of Silkworm Genome Biology, College of Sericulture, Textile and Biomass sciences, Southwest University, Chongqing 400716, China.

2. Cancer Centre, Reproductive Medicine Centre, Medical Research Institute, Southwest University, Chongqing 400716, China

3. NHC Key Laboratory of Birth Defects and Reproductive Health (Chongqing Key Laboratory of Birth Defects and Reproductive Health, Chongqing Population and Family Planning Science and Technology Research Institute), Chongqing 400020, China

4. Chongqing Engineering and Technology Research Center for Silk Biomaterials and Regenerative Medicine, 400716 Chongqing, China.

5. The Ninth People's Hospital of Chongqing, Affiliated Hospital of Southwest University.

* First authors

Changhong Li and Kui Zhang contributed equally to this work.

\# Corresponding author

Liqun Yang, State Key Laboratory of Silkworm Genome Biology, Southwest University, Chongqing 400716, China. Tel.: +86 23 68251712; fax: +86 23 68251128. Address: \#2, Tiansheng Rd., Beibei District Chongqing, China. Email address: cysylq@,swu.edu.cn

\section{Conflict of interest}

No potential conflicts of interest were disclosed.

Simple Summary: Dehydrodisoeugenol (DEH), as one of the components of Nutmeg, has been reported to have an excellent therapeutic effect on digestive system disorders. However, most of these reports are limited to displaying the composition and activity of DEH. The specific molecular mechanism has barely been explored. In this study, we used DEH to treat colorectal cancer cells innovatively. Surprisingly, we found that DEH has a strong inhibitory effect on cell proliferation and growth of colorectal cancer cells, showing an excellent antitumor activity in vivo and in vitro. As far as we know, this is the first report of an anti-tumor role of DEH in colorectal cancer and our data strongly suggests that DEH may be a promising therapeutic agent against colorectal cancer in the future.

Abstract: Dehydrodiisoeugenol (DEH), a novel lignan component extracted from the Nutmeg seeds, displays noticeable anti-inflammatory and anti-allergic effects in digestive system diseases. However, the mechanism of its anti-cancer activity in 
gastrointestinal cancer is still to be investigated. Here, the anti-cancer effect of DEH to human colorectal cancer and its underlying mechanism were evaluated. The DEH treatment arrests the cell cycle of colorectal cancer cells at G1/S phase, which leading to a significant cell growth inhibition. Moreover, it can induce strong cellular autophagy and the autophagy would be inhibited through autophagic inhibitors with reducing EDH-induced inhibition of cell growth in colorectal cancer cells. Further studies indicated that DEH can also induce endoplasmic reticulum (ER) stress, and could subsequently stimulating autophagy through activating PERK/eIF2 a and IRE1 a /XBP-1s/CHOP pathways. Knockdown of PERK or IRE1 $\alpha$ can significantly decrease the DEH-induced autophagy and retrieve cell viability in cells treated with DEH. What's more, DEH exhibits significant anti-cancer activities through CDX- and PDXmodel as well. Taken together, our studies strongly suggest that DEH might be a potential anti-cancer agent against colorectal cancer via activating ER stress-induced autophagy inhibition.

Keywords: Colorectal cancer, Dehydrodiisoeugenol (DEH), Autophagy inhibition, Endoplasmic reticulum (ER) stress, anti-cancer agent

\section{Introduction}

Colorectal cancer (CRC), including colon cancer and rectal cancer, is one of the most common cancers in the world at present[1,2]. It is the third most common health problem as well as the fourth leading cause of mortality world widely[3,4]. Besides, colorectal cancer is the third most common cancer in male and the second most common cancer in female[5,6]. Since its early symptoms are not obvious, most patients are at an advanced stage when they were diagnosed[7,8]. The colorectal cancer not only hurts the digestive system, but also acutely impairs the lymph, liver, lung, and bone[9,10]. Although the current treatments such as chemotherapy, radiotherapy, and surgery were used widely, the prognosis of patients with colorectal cancer is awful[11,12]. More terribly, the absorption function of the intestine will be reduced particularly due to the faultiness in the intestinal mucosa[13]. This situation leads to diarrhea, abdominal pain and other adverse symptoms that affect the normal life of the patients eventually[14,15].

With the development of the screening of anti-tumor drugs, more and more monomer compounds from the traditional Chinese medicine have been explored to play an considerable roles in the treatment of a variety of tumors[16-18]. Hence, it is meaningful to explore more effective and nontoxic drugs for colorectal cancer treatment. 
In addition, this strategy would be a fantastic way to improve the life quality and therapeutic effect of patients[19-21].

Nutmeg, an evergreen tree plant, has been widely used as a traditional Chinese medicine, whose pharmacological effects are aimed at curing the discomfort of digestive tract, abdominal distending pain, and persistent diarrhea[21,22]. Dehydrodiisoeugenol (DEH), as a compound extracted from Nutmeg seed, has also been proved to have many characteristics such as liver protection, anti-thrombotic, antiallergic, anti-oxidant and anti-tumor[23-25]. But the anti-cancer effect of DEH on colorectal cancer and the inhibition mechanism of that are remain unclear. In this study, the anti-tumor activity of DEH on colorectal cancer were investigated through both cellderived xenograft (CDX) and patient-derived tumor xenograft (PDX) model. Our results showed that DEH may be a promising therapeutic implication for the treatment of colorectal cancer.

\section{Results}

\subsection{DEH inhibits proliferation of colorectal cancer cell in vitro}

Dehydrodiisoeugenol (DEH, CAS : 83377-50-8), known as Isoeugenol or Diisoeugenol, is a kind of benzofuran-type neolignane extracted from Myristica fragrans Houtt, which has been prescribed in Chinese medicine for a long time[33]. The structural formula of DEH was shown in Figure S1A. To explore the effect of DEH on cancer cells and normal cells, an MTT assay was used to determine the 50\% inhibitory concentration $\left(\mathrm{IC}_{50}\right)$ of $\mathrm{DEH}$ on colorectal cancer cells. Here, human normal normal colon epithelial cell NCM460 and two types of colorectal cancer cells, including HCT 116 and SW620, were investigated. All the detected cells were incubated with a series of different concentrations of DEH (0, 0.001, 0.01, 0.1, 1, 10, 20, 40, 60, 80, 100, 125, and $1000 \mu \mathrm{M}$ ) for $48 \mathrm{~h}$. The result showed that DEH can significantly inhibit cells growth at a relatively low concentration. What's more, the medial lethal concentration of NCM460 is significantly higher than that in HCT 116 and SW620 cells. The $\mathrm{IC}_{50}$ of DEH in HCT 116 and SW620 cells were $54.32 \mu \mathrm{M}$ and $46.74 \mu \mathrm{M}$, respectively (Figure 1A). To interpret the anti-cancer effect of DEH on colorectal carcinoma cells in depth, HCT 116 and SW620 cells were incubated DEH with a series of different concentrations $(0,10,20,30,40,50,60,70$ and $80 \mu \mathrm{M})$ for $24 \mathrm{~h}, 48 \mathrm{~h}$ and $72 \mathrm{~h}$ respectively. The results showed that $\mathrm{DEH}$ can inhibit colorectal carcinoma cell lines HCT 116 and SW620 in a time- and dose-dependent manner (Figure 1B). After 
incubating with DEH, cell numbers decreased in a dose-dependent manner and the morphology of DEH-treated cells changed distinctly (Figure 1C). Furthermore, EDU staining assay was implemented to detecting cell proliferation of the detected cells. The results showed a notable decrease at the percentage of EDU positive cells in cells incubated with DEH compared with DMSO treated groups (Figure 1D). The cell activity was evaluated by plate clone formation assay, and the results showed that DEH can significantly inhibit the detected cells activities in a dose-independent manner (Figure S1B). Soft agar assays were used to investigate the self-renewal capability of HCT 116 and SW620 cell lines that treated different concentrations of DEH $(0,20,60$ $\mu \mathrm{M})$ for $48 \mathrm{~h}$. Results showed that the colonies were smaller and fewer at number in the DEH-treated groups compared to the control groups (Figure 1E). Taken together, these data indicated that DEH could remarkably suppress the cell proliferation of colorectal cancer cells with a dose-dependent manner in vitro.

\subsection{DEH inhibits cell growth by inducing cell cycle arrest at the G1 phase}

To explore the reason about DEH inhibition to cell growth and proliferation, the flow cytometry was used to examine the cell cycle of detected cells. There was an approximately $30 \%$ increase in the number of cells at G1 phase among the DEH-treated cells compared with control cells both in HCT 116 and SW620 cells (Figure 2A). The results revealed that DEH treatment caused distinct accumulation of cells in the G1/S phase. It indicated that DEH inhibits cell growth and proliferation by inducing cell cycle arrest at the G1 phase. To further confirm the alterations, we detected the expression levels of p21, CDK2, CDK4, Cyclin D1, Cyclin E1 and Cyclin E2 proteins, which can promote cells passing through the G1/S checkpoint. The results showed that the proteins expression of related cyclins and CDKs in DEH-treatment cells was significantly reduced compared with counterparts in control group cells in a dose-dependent and time-dependent manner (Figure 2B-C). Whether the decline of cell viability is caused by apoptosis in some extent? We measured the apoptosis rate in cells by flow cytometry again. Nevertheless, the results showed that there is no obvious apoptosis appeared in cells after DEH treatment (Figure S1C). These data manifested that DEH inhibits cell growth by inducing cell cycle arrest rather than apoptosis in colorectal cancer cells.

\subsection{DEH induces autophagy in the colorectal cancer cells}

To determine whether DEH induced cellular autophagy in colorectal cancer cells, an immunofluorescence assay was performed to check the distribution of LC3B, which 
is widely considered as a marker of autophagy. The LC3B-positive cells were increased remarkablely in the cells incubated with $\mathrm{DEH}$, compared with the control group both in HCT 116 and SW620 cells. The numbers of LC3B puncta per cell were also significant increased after DEH treatment (Figure 3A). The autophagy vesicle formation was also analyzed by using GFP tagged MAP1LC3B expression system. GFP-MAP1LC3B-positive cells were significant increased after incubating with DEH, compared with the control group (Figure 3B). The autophagic vesicles were eventual become to autophagosomes observed by transmission electron microscopy (TEM). As shown in Figure 3C, abundant autophagosomes were observed in HCT 116, and SW620 cells after incubating with DEH. The protein expression level of LC3B, and ATG7 were definitely upregulated after DEH treatment in a dose- and time-dependent manner (Figure 3D, Figure S2B). Noticeably, as the hallmark of autophagy, p62 was remarkable increased after DEH treatment. It implies us that the DEH-induced autophagy in colorectal cancer was disturbed by some actions.

\subsection{DEH treatment inhibits the autophagic flux}

The accumulation of LC3B puncta might result from the autophagy flux blockage. The p62 protein was increased remarkablely after cells incubated with $\mathrm{DEH}$, it implies that DEH-induced autophagy may be obstructed by the autophagic flux inhibition convincingly. To further conform that, a GFP-RFP-LC3 double label vector system was used in the next step. As shown in Figure 4A, GFP and RFP were almost uniformly distributed in the DMSO-treated cells, due to low level of background autophagy. When incubated with EBSS, the RFP puncta dominates at the starvation treated cells. While the cells treatment with $\mathrm{CQ}$, which can block the fusion of autophagosomes with lysosomes and widely used as a classical inhibitor of cellular autophagic flux, both GFP and RFP puncta were almost completely coincident. The results of those control groups show clearly that GFP-RFP-LC3 double label vector system was an effective tool to evaluate the autophagic flux. When the cells incubated with $\mathrm{DEH}$, yellow puncta dominate in detected cells, both in HCT 116 and SW620 cells. When the cells together incubated with DEH and 3-methyladenine (3-MA), which is a selective inhibitor of PI3K and can inhibit the formation of the early-stage autophagosome, the expression level of LC3B-II was decreased compared with DEH-treatment alone (Figure 4B). The expression level of LC3B-II was also analyzed after cells incubated with DEH together with or without CQ and Baf A1. The two compounds can inhibit the fusion of autophagosomes with lysosomes and widely used as autophagic flux inhibitor. DEH, 
$\mathrm{CQ}$, and Baf A1 can cause the accumulation of LC3B-II alone, while DEH together incubated with CQ or Baf A1 could not cause the further increase of accumulation level of LC3B-II, compared with DEH-treated alone (Figure 4C). In summary, these results demonstrated that DEH could be acted as an autophagy flux inhibitor to cause the accumulation of autophagosomes.

\subsection{DEH induces ER stress in the colorectal cancer cells}

The above results demonstrated that DEH could inhibit colorectal cancer cell proliferation and trigger cellular autophagy via impairing the autophagic flux. Besides, we hav observed that DEH treatment could induce the cellular vacuolization formation in cells treated with DEH. Then, the transcriptome analysis was performed to investigate the changes of gene expression pattern in cells that treated with DEH for 48 h. As shown in Figure 5A, unfolded protein response (UPR)- related factors were enriched keenly in cells DEH treated through GESA website. These genes related to ER stress, PERK and IRE1 a pathways were upregulated at the transcriptional level especially both in HCT 116 and SW620 cells (Figure 5B). To confirm this phenomenon at the cellular level, transmission electron microscopy was used to observe the DEHtreated colorectal cancer cells intensively. The results showed that more dilated cytoplasmic vacuoles which were recognized as dilated ER lumens. The numerous ER stress were identified in DEH-treated colorectal cancer cells than the control group distinctly (Figure 5C). To verify these results, we further examined ER-stress-related proteins, including BiP, Ero1-L $\alpha$, PERK, eIF2 $\alpha$, p-eIF2 $\alpha$, IRE1 $\alpha$, XBP-1s and CHOP, in colorectal cancer cells treated with different concentrations of DEH for $48 \mathrm{~h}$. The results showed that the expression level of examined ERS-related proteins were increased in a dose-and time-dependent manner (Figure 5D, Figure S2C). Taken together, these data demonstrated that DEH could significantly induce ER stress in human colorectal cancer cells.

\subsection{DEH-induced autophagy is related to the ER stress-dependent activation of PERK and IRE1 $\alpha$}

ER stress is closely related to the activation of autophagy[34]. To clarify the correlation between ER stress and autophagy induced by DEH, RNA knock-down experiments were performed. The inhibitory effect of DEH on shIRE1 $\alpha$-cells and shPERK-cells was weaker than that of GFP-group notably (Figure 6A). A contactdependent proliferation assay by plate cloning formation was performed to further 
confirm the effect of DEH on cells with the gene of IRE1 $\alpha$ and PERK interfered compared with control group. These results confirmed that DEH-induced ERS was damaged to colorectal cancer cells (Figure 6B ). Then we checked it at the protein level, western blot assays showed the expression level of IRE1 $\alpha$ and LC3B.Tubulin was used as the control. The data showed that the expression of LC3B-II protein was decreased after DEH treatment with shIRE1 $\alpha$ compared to control group (Figure 6C). Subsequently, we used the inhibitor of IRE1 $\alpha$ to further identify the the role of IRE1 $\alpha$ played in DEH-induced autophagy (Figure 6D-E). The results were consistent with the gene interference treatment. Above findings showed that DEH could induce ER-stress through unfolded protein responses, and might cause the autophagy inhibition by activating PERK and IRE1 $\alpha$ pathways subsequently.

\subsection{DEH exerted anti-cancer efficacy in vivo}

To detect the effects of DEH about the tumorigenic ability in colorectal cancer cells, we performed subcutaneous tumor experiment by using cell-derived xenograft (CDX) and patient-derived tumor xenograft (PDX) model in vivo. The tumor size, weight and growth rate were all significantly decreased in the DEH-treated group, compared with the control group (Figure 7A, Figure S3A). Furthermore, the rate of the Ki67-positive cells was significantly reduced in DEH-treated group compared to the control group (Figure 7C, Figure S3B). It implies that DEH also inhibited the tumor growth in vivo. Importantly, DEH had no remarkable effect on the body weight of all the detected mice (Figure S3C). Next, we examined the heart, liver, spleen, lung, and kidney of mice that treated with DEH or DMSO by H\&E staining. Apparently, DEH treatment has no significant effect on the pathological features of mice organs of measuring mice (Figure 7D), which indicated that the drug is safe in mice. Moreover, H\&E staining was used to detect the tumors from DEH treatment group and control group (Figure 7B). These results showed that DEH have barely toxic and side effect to experimental mice. Meanwhie, the magnitude of tumors was decreased majorly in DEH treatment group. All above data have been proved that DEH has a significant inhibitory effects on the tumorigenesis and the growth of colorectal cancer with low-toxicity.

\subsection{DEH induces ER stress and autophagy flux inhibit in vivo}

Next, we ground the tumor from injected mice and detected the related proteins by western blot assays again. The results showed that the expression level of ER-stressrelated proteins, including BiP, PERK and IRE1 $\alpha$ wree upregulated in colorectal cancer 
after DEH-treated continuously (Figure 7E). The expression level of autophagy related proteins in tumor tissues was also increased significantly after treated with DEH compared with control group (Figure 7F). Thoese results at individual level are consistent with the cellular level greatly. Taken together, these findings implied that DEH-induced autophagy might be disturbed by the DEH-induced ER-stress substantially, which is indicated that DEH also displays the excellent anti-tumor effect in vivo utterly.

\section{Discussion}

Despite numerous therapeutic and screening attempts are emerging, colorectal cancer still remains a major life-threatening malignancy[35]. Unfortunately, patients with advanced or recurrent colorectal cancer usually have a low survival rate due to drug toxicity of chemotherapy or side-effect of radiotherapy after the common treatment for colorectal cancer[36]. develop the effective therapeutic drugs for Therefore, it is of great urgency to actively explore the high-efficiency and low-toxicity drugs for patients after surgery with poor prognosis to improve the life quality of them[37]. At present, the treatment of diseases with traditional Chinese medicine has been shown great potential. As we all known, the Chinese traditional medicine has a long history in the treatment of many diseases and the research on monomer isolated from traditional Chinese medicines has also become more and more prevalent[38]. Moreover, the traditional Chinese medicine is a potential way for the treatment of cancer in the light of its extremely low toxicity during its long course of application[39]. Nutmeg, as an evergreen tree plant, is such a traditional Chinese medicine, whom has proven its potential ability in the treatment of gastric cancer, lung cancer, skin tumors, melanoma, osteosarcoma and leukemia[40]. DEH, a kind of highest-acquired active monomer, is extracted from the seeds of Nutmeg. Recent studies showed that DEH exerts fantastic anti-inflammatory and anti-allergic effects in various digestive disorders[41]. However, the research on the effect of DEH in colorectal cancer has not been reported.

Here, we described the critical role of DEH in the treatment of colorectal cancer and explored the relevant mechanisms for the possible therapeutic application. In this study, we evaluated the anti-tumor activity of DEH in colorectal cancer. First of all, we demonstrated that DEH significantly inhibited cell proliferation and growth in a doseand time-dependent manner in vitro through MTT and EDU assays. Soft agar assay 
showed that the colony size became lesser and smaller after DEH treatment. In addition, the flow cytometry analysis indicated that DEH inhibited cell growth in a time- and dose-dependent manner by arresting cell cycle at G1/S phase. What's more, the results showed that DEH could in no shape induce apoptosis in colorectal cancer cell.

Based on our understanding of autophagy's physiological function, we now know that the basic level of autophagy and the stress-induced moderate autophagy may be important to maintain the cell metabolism[42,43]. However, there is no definite evidence of autophagy's specific role and physiological function in different cell microenvironment. Besides, the unreasonable autophagy or autophagy flux blocked exceptionally will reduce the ability of clearing intracellular proteins and damaged organelles, resulting in genomic instability, thus inhibiting cell proliferation and growth[44-46]. In our study, the results showed that DEH could significantly induce autophagy in colorectal cancer cells. TEM obersevations also confirmed this. In order to further explore the mechanism, we detected the expression of autophagy related protein. p62 protein was up-regulated by $\mathrm{DEH}$ treatment, which implied that $\mathrm{DEH}$ induced autophagy blocking has a devastating effect on cells, and the reason of autophagy inhibition need to be explored in depth. Then GFP-RFP-LC3 double label vector system was used to detect the flow of autophagy induced by DEH. The results confirmed that autophagy flow was indeed blocked. It is suggested that the autophagy induced by DEH may due to the impaired degradation process of autophagosomes. Next, we combined with the corresponding autophagy activator and inhibitor to detect the protein of DEH by western blot. The results confirmed that the autophagy inhibition may be occured in the later stage of the autophagy. Blocking the autophagic flow of activated autophagy helps to inhibit the growth and proliferation of cells that should die, such as tumor cells receiving chemotherapy. This made an autophagy-inhibition strategy to treat cancer become possible for cancer cell. This may be due to the enhanced autophagy of proteins. Finally, DEH can inhibit the growth of colorectal cancer cells further through autophagy inhibition.

In our study, microscopic observation and transcriptome data analysis showed that DEH also caused strongly endoplasmic reticulum stress. Then the transmission electron microscope observation combined with western blotting assay were used to confirm that DEH did cause endoplasmic reticulum stress in colorectal cancer cells in dose-and time-dependent manner. According to the previous reports, endoplasmic reticulum stress not only can increase autophagy but also can make autophagy inhibition[47,48]. 
Besides, endoplasmic reticulum stress-mediated autophagy which have been shown that UPR and its downstream pathways may play an important role in has been widely indentified.

In order to elucidate this signaling pathway, we interfered the two key genes of endoplasmic reticulum stress pathway, and then treated cells with drugs again. Through MTT and plate cloning experiments, we found that the autophagy inhibition induced by DEH was activated by DEH-induced endoplasmic reticulum stress. However, the process of DEH induced endoplasmic reticulum stress activate the autophagy inhibition remains to be further explored.

Subsequently, the xenograft experiments with CDX and PDX model were used to check the anticancer effect of DEH at the individual level. The growth of tumors formed in nude mice subcutaneously grew slowly after DEH treatment with the weight and volume of the tumors formed in nude mice were significantly slower and smaller after DEH treatment. But the weight and volume of mice were barely varied. These results suggest that DEH can inhibit the growth of colorectal cancer in vivo and in vitro even at individual level. What's more, the toxicity of DEH is low and is safe in mice. In addition, we also detected the expression level of autophagy related proteins and endoplasmic reticulum stress-related proteins in tumors from detected mice. The results were consistent with the cellular level. Accordingly, our study about DEH may provide a promising therapeutic agent for the treatment of colorectal cancer. Taken together, our study has demonstrated that clearly: On the one hand, DEH could inhibit the cell proliferation and cell growth. On the other hand, DEH could induce damage ERSautophagy to realize the obvious anticancer effect to colorectal cancer (Figure 8). This role of DEH played in colorectal cancer maybe a new strategy to patients. Moreover, according to the previous report, many clinical chemotherapy drugs and traditional Chinese medicines inhibit cell proliferation and growth by inducing apoptosis to cause cell death[49]. Fortunately, it's no doubt that DEH has a wonderful anti-cancer activity on colorectal cancer with a lower toxicity.

\section{Materials and methods}

\subsection{Cell culture}

Human colorectal cancer cell lines (HCT 116 and SW620) and human embryonic renal cell lines 293 FT were purchased from the American Type Culture Collection (ATCC, USA) and stored in our laboratory. HCT 116 cells were cultured in McCoy's 
5A medium (Gibco, USA), containing $10 \%$ fetal bovine serum (FBS, Gibco) with $1 \%$ penicillin-streptomycin $(\mathrm{P} / \mathrm{S} ;$ Invitrogen, USA). SW620 cells were cultured in Dulbecco's Modified Eagle Medium (DMEM; Gibco, USA) added with $10 \%$ and $1 \%$ penicillin and streptomycin (P/S). 293 FT cells were cultured in DMEM with $10 \%$ FBS and $1 \% \mathrm{P} / \mathrm{S}$ maintaining with $1 \% \mathrm{G} 418$ (Invitrogen, USA), added $2 \%$ glutamine (Invitrogen, USA), $1 \%$ non-essential amino acids (Invitrogen, USA) and $1 \%$ sodium pyruvate (Invitrogen, USA). All cells were cultured under standard condition at $37{ }^{\circ} \mathrm{C}$ in a humidified atmosphere containing $5 \% \mathrm{CO}_{2}$.

\subsection{Drug treatment}

Dehydrodiisoeugenol (DEH, CAS No. 2680-81-1) was purchased from Chengdu Herbpurify CO., LTD and dissolved in Dimethyl Sulfoxide (DMSO) as $400 \mathrm{mM}$ stock solutions. DEH was diluted to corresponding culture medium or PBS for in vitro and in vivo experiments. HCT 116 and SW620 cells were treated with DEH in different concentration $(0,20,40$, and $60 \mu \mathrm{M})$. Microscopy (Olympus, Japan) was used to detect the cell morphology. Simultaneously, hemocytometer was used for counting cell numbers. Every experiment was repeated at least three times independently.

\subsection{Cell viability and proliferation assays}

The cell viability and cell proliferation detections of CRC cells treated with DEH were determined by 3-(4, 5-dimethylthiazol-2-yl)-2,5-diphenyltetrazolium bromide (MTT) (Sigma Aldrich, USA) assay[26]. Briefly, cells were counted in logarithmic phase at $1.5 \times 10^{3} /$ well and then seeded in 96 -well plates with medium $(200 \mu \mathrm{L})$ and attached overnight before the start of treatment. Then cells treated with DEH in different concentrations $(10,20,30,40,50,60,70$, and $80 \mu \mathrm{M})$, Culture medium containing DMSO or DEH was added to each well evenly (DMSO treatment as the control group). At the specific time points, cells were incubated with MTT $(5 \mathrm{mg} / \mathrm{mL}, 20 \mu \mathrm{L} /$ well $)$ for $2.5 \mathrm{~h}$. The medium containing the formazan was then removed and instantly replaced by DMSO $(200 \mu \mathrm{L})$ for solving the formazan complex. Microplate reader (Thermo Fisher, USA) was used to measure the absorbance at a wavelength of $560 \mathrm{~nm}$. According to the $\mathrm{OD}$ value, the $\mathrm{IC}_{50}$ and cell viability of cells was valuated via analyzing by GraphPad software. Then, cells were treated with DEH at 0, 20,40 and 60 $\mu \mathrm{M}$ for 24,48 , and $72 \mathrm{~h}$, with the method as described above. Each experiment was performed in triplicates independently. 


\subsection{EDU staining}

The cell's ability to proliferation was measured by using Click-iT ${ }^{\circledR}$ EdU Imaging Kits (Invitrogen) according to the instruction and operation manual. Briefly, $4 \times 10^{4}$ cells were seeded in 24-well plates. The adherent cells were incubated with different concentrations of DEH $(0,20,40$, and $60 \mu \mathrm{M})$ for 2 days. Then, cells were incubated with $10 \mu \mathrm{M}$ EDU (Sigma Aldrich, USA) for $40 \mathrm{~min}$. After fixed in $3.7 \%$ PFA in PBS for $15 \mathrm{~min}$, cells were incubated with $3 \%$ bovine serum albumin (BSA) and subsequently permeabilized in $0.5 \%$ Triton X-100. Cells were incubated with Click$\mathrm{iT}^{\circledR}$ reaction cocktails for 40 minutes at room temperature and protected from light. Nuclei was stained by DAPI for $30 \mathrm{~min}$ at room temperature. The fluorescence images were obtained by using a fluorescent inverted microscope, and the percentage of EDUpositive staining was calculated from 10 microscopic fields randomly.

\subsection{Plate clone formation and soft agar assays}

The cell activity and proliferation were detected by plate clone formation assay. Briefly, 800 cells were plated in 6 -well plates. The adherent cells were incubated with different concentrations of DEH for 2 weeks. Then samples were stained with $0.1 \%$ crystal violet and captured via a digital scanner. The effect of DEH on the cell selfrenewal and colony formation ability in HCT 116 and SW620 cells was determined by soft agar assay[27,28]. Briefly, $1.5 \mathrm{~mL}$ of basic medium containing $0.6 \%$ low melting agarose and indicated concentrations of DEH was added to well culture-plates. And then, replenishing $1 \mathrm{~mL}$ complete medium, which containing $0.3 \%$ low melting agarose, 800 cells and indicated concentrations of DEH, was added to the top of the solidified top layer. The colonies were observed and imaged in an invert microscope. Finally, all of the clones were stained with MTT at $37{ }^{\circ} \mathrm{C}$ for $30 \mathrm{~min}$ and captured by a digital scanner. The number of colonies were visualized and quantified simultaneously.

\subsection{Flow cytometry}

Cells were put into cell cultured plate and cultured under normal conditions. The adherent cells were incubated with cell-culture medium containing different concentrations of DEH for 2 days for cell cycle and apoptosis analysis respectively. For the cell cycle assay, cells were harvested and fixed in cold $75 \%$ ethanol at $4{ }^{\circ} \mathrm{C}$ overnight. After washing with PBS to remove the residual alcohol, cells were incubated with propidium iodide (PI, BD, USA) and RNase A (Sigma Aldrich, USA) at $37^{\circ} \mathrm{C}$ for 
$1 \mathrm{~h}$. For apoptosis assay, cells were harvested and washed with cold PBS. And then, cells were resuspended in $100 \mu \mathrm{L}$ binding buffer (BD, USA). Next, the cells were incubated with FITC-labelled Annexin V (BD, San Jose, CA, USA) and PI at room temperature for 15 min. All samples were analyzed by the FACS C6 (BD, USA) with Cell Quest software.

\subsection{Western blot analysis}

The western boltting was performed to detect the protein expression level or protein phosphorylation as described in our previous study[28,29]. Briefly, colorectal cancer cells (HCT 116 and SW620) were harvested, washed with cold PBS buffer and then suspended in RIPA lysis buffer (Beyotime, China) contain with phosphatase inhibitor sodium fluoride (Beyotime, China) and phenylmethylsulfonyl fluoride (PMSF, Beyotime, China). Cell lysates were denatured at $100{ }^{\circ} \mathrm{C}$ for 25 minutes, and protein concentrations were detected by the BCA protein assay kit (Beyotime, China). Total of $45 \mu \mathrm{g}$ proteins were subjected to $10 \%$ sodium dodecylsulphate polyacrylamide gel electrophoresis (SDS-PAGE). The protein bands were transferred to PVDF membranes (Millipore, USA) by the Trans-Blot Turbo transfer system (Bio-Rad, USA). After being blocked with $5 \%$ bovine serum albumin (BSA) in TBST buffer at room temperature for $2.5 \mathrm{~h}$ with shake gently. The PVDF membranes were gently incubated with antibodies against human Tubulin (1: 5 000, AF1216, Beyotime, China[[zk1])[zk2], p21 (1:1 000, \#2947, Cell Signaling Technology, USA), CDK2 (1:1 000, \#2546, Cell Signaling Technology, USA), CDK4 (1:1 000, \#12790, Cell Signaling Technology, USA), Cyclin D1 (1:1 000, \#2978, Cell Signaling Technology, USA), Cyclin E1 (1:1 000,\#4129, Cell Signaling Technology, USA), Cyclin E2 (1:1 000; \#4132, Cell Signaling Technology, USA), BiP (1:1 000, \#3177, Cell Signaling Technology, USA), Ero1-La (1:1 000, \#3264, Cell Signaling Technology, USA), PERK (1:1 000; \#5683, Cell Signaling Technology, USA), eIF2 $\alpha$ (1:1 000, \#5324, Cell Signaling Technology, USA), peIF2 $\alpha$ (1:1000, \#3398, Cell Signaling Technology, USA), IRE1 $\alpha$ (1:1 000, \#3294 Cell Signaling Technology, USA), XBP-1s (1:1 000; \#47134, Cell Signaling Technology, USA), CHOP (1:1 000, \#2895, Cell Signaling Technology, USA), LC3B (1:1 000; \#3868, Cell Signaling Technology, USA), p62 (1:1 000, \#88588, Cell Signaling Technology, USA) and ATG7 (1:1 000; \#8558, Cell Signaling Technology, USA) were incubated at $4{ }^{\circ} \mathrm{C}$ overnight. After washing with TBST buffer, the samples were incubated with HRP-conjugated secondary antibodies (1:10 000, Life Technology, USA) for $2.5 \mathrm{~h}$ at room temperature. Finally, proteins bands were visualized via capturing 
with the detection analysis system (Clinx Science, China).

\subsection{Transcriptome data analysis and methodology}

The colorectal cancer cells were incubated with or without DEH for 2 days, then cells were collected and submitted to Novogene (Beijing, China) for transcriptome sequencing and analysis. The row data of RNA-sequence were analyzed and evaluated by GESA database[30,31]. Next, we filtered the data and cleared up the untrusted data to cluster the differentially expressed genes. We classified these genes to determine the expression patterns of different genes under different experimental conditions for exploring the transformation of the signaling pathway which caused by DEH treatment. After classification, the gene difference analysis was conducted again.

\subsection{Electron microscopy}

Transmission electron microscopy was used to identify the ultrastructure of endoplasmic reticulum and autophagosome[30]. HCT 116 or SW620 were put into cell cultured plate. When cell density was reached up to $40 \%$, cells were incubated with or without $60 \mu \mathrm{M} \mathrm{DEH}$ for 2 days at $37{ }^{\circ} \mathrm{C}$. Collected cells were fixed in in $2.5 \%$ glutaraldehyde (Sigma-Aldrich, G5882) for 2 days at $4{ }^{\circ} \mathrm{C}$. Samples were detected and analyzed by Wuhan Microscopic Biotechnology Co., Ltd. The images were processed by Adobe photoshop CS6 and Adobe Illustrator CS6.

\subsection{Immunofluorescence assay}

Cells were collected and fixed by cold-methanol. After blocking with $10 \%$ BSA in PBS at $37{ }^{\circ} \mathrm{C}$, the cells were incubated with an LC3B (D11) XP® Rabbit mAb (1: 500; CST, USA) at $4{ }^{\circ} \mathrm{C}$ overnight. Cells were then incubated with Alexa Fluor labeled secondary antibody (1: 2 000; Invitrogen, USA) for 488-conjugated goat anti-rabbit and 594-conjugated goat anti-mouse at room temperature for $2 \mathrm{~h}$. DAPI (1: 500,) was then used to stain the nuclei, and LC3B positive cells were captured by an Olympus FV1000 confocal fluorescence microscope[32].

\subsection{Tumor xenografts}

Five-week-old female NOD/SCID mice were used for animal study. The xenograft mice models including HCT 116, SW620, and patient-derived xenograft (PDX) were grouped randomly. HCT 116 and SW620 cells $\left(1 \times 10^{6}\right.$ cells $)$ and the patient-derived xenograft (PDX) model in $100 \mu \mathrm{L}$ of PBS were injected into both flanks of each mouse subcutaneously. When the tumors developed into a certain volume, DEH was used as 
follow: The control group $(\mathrm{N}=3)$ was injected intraperitoneally with $100 \mu \mathrm{L}$ PBS (contained $0.1 \% \mathrm{DMSO}$ ); the other groups ( $\mathrm{N}=3$ per group) were injected with $\mathrm{DEH}$ at $40 \mathrm{mg} / \mathrm{kg}$ (mice body weight) once every other day. Tumor volume was also measured once every 2 days. At the end, tumors were removed from the body of mice. We photographed and weighed the tumors instantly. All animal experiments were approved by the Experimental Animal Care and Use Committees of the Institute of Sericulture and Systems Biology and Institutional Animal Care and Use Committees of the Southwest University, Chongqing, China (Approval number: 2019-016).

\subsection{IHC and H\&E assays}

The xenograft tumors were fixed in $3.7 \%$ paraformaldehyde (PFA) at $4{ }^{\circ} \mathrm{C}$ for 48 hours. After washing with PBS, samples were dehydrated and embedded in paraffin. The paraffin sections were deparaffinized following hydration and antigen retrieval. The sections were incubated in $0.3 \%$ hydrogen peroxide for 15 minutes and subsequently incubated with Ki67 (8D5) Mouse mAb (1:200, \#9449, Cell Signaling Technology, USA), BiP (C50B12) Rabbit mAb (1:100, \#3177, Cell Signaling Technology, USA) or LC3B (D11) Rabbit mAb (1:100, \#3868, Cell Signaling Technology, USA) at $4^{\circ} \mathrm{C}$ overnight, respectively. After washing with PBS, the sections were incubated with anti-rabbit biotinylated antibody (Cell Signaling) at room temperature for $2 \mathrm{~h}$. The amplified positive signal was observed under the microscope, performing with DAB reagent (Beyotime, China). The rates of Ki67 positive signal were calculated from five randomly selected fields and quantified the Ki67-positive cells subsequently. In addition, the tissues and tumors were stained with hematoxylin and eosin (H\&E) (Sangon, China). All the images were obtained by microscopy.

\subsection{Statistics Analysis}

All experiments were carried out with three independent technical and biological replicates at least. Flow cytometry study results were analyzed by FlowJo software according to the software operation instruction. Statistical analysis was performed by using Graphpad Prism. All the quantitative data acquired were presented as mean \pm standard deviation (SD). Significant different computation come from Unpaired Student's t-test, and p-value $<0.05$ were considered as statistically significant. ${ }^{*}$ p $<$ $0.05, * * \mathrm{p}<0.01$, and $* * * \mathrm{p}<0.001$.

\section{Conclusions}


In summary, our data unravel a novel anticancer mechanism of DEH in human colorectal cancer by inducing cell cycle arrested in a dose- and time-dependent manner and causing autophagy inhibition via ER stress, which could inhibit the growth of colorectal carcinoma more effectively. These findings suggest that DEH may be a promising therapeutic drug for the treatment of colorectal cancer.

\section{Figure legends}

Figure.1 DEH inhibits the growth of colorectal cancer cells in vitro. A. Colorectal carcinoma cells, including NCM460, HCT 116 and SW620, were incubated with a series of different concentrations of DEH for $48 \mathrm{~h}$. Cell viability was measured by MTT assay. The values of $\mathrm{IC}_{50}$ of DEH for $48 \mathrm{~h}$ in detected cells are marked in the lower-left corner. B. Dose-and time-dependent effects of DEH on HCT 116 and sw620 cells. The cells were incubated by DEH at various concentrations for 24,48 , and $72 \mathrm{~h}$. Cell viability was measured by MTT assay. The results are represented as the means \pm SD $(n=3)$. C. Cell morphology of HCT 116 and sw620 cells after incubating with indicated concentration of DEH or DMSO for $48 \mathrm{~h}$. Scale bar, $10 \mu \mathrm{m}$. The histograms represent the effect of EDH on the cell viability. D. Images and quantification of HCT 116 and SW620 cells positive for EDU staining after treatment with DEH for $48 \mathrm{~h}$. Scale bar: $100 \mu \mathrm{m}$. E. Colony formation was investigated by soft agar assay after incubating with DMSO, 20, or $60 \mu \mathrm{M}$ DEH, Scale bar: $15 \mu \mathrm{m}$. Colony number was counted, and the statistical data are shown as the mean $\pm \mathrm{SD}$. The notability analysis was performed by the Unpaired Student's t-test, and $p$ value less than 0.05 was considered significant. $* p$ $<0.05, * * \mathrm{p}<0.01, * * * \mathrm{p}<0.001$.

Figure 2. DEH inhibits cell growth by arresting cell cycle at the G1/S phase. A. Cell cycles of HCT 116 and SW620 cells were investigated via flow cytometry after treatment with or without DEH for $48 \mathrm{~h}$. The distribution ratio of G1, S, and G2 of panel A were counted. B. Western blotting assays were performed to detect the expression of p21, CDK2, CDK4, Cyclin D1, Cyclin E1, Cyclin E2, and Tubulin in HCT 116 and SW620 cells after treatment with DEH and the densitometry of Western blotting bands of panel C. The protein expression levels of p21, CDK4, Cyclin D1, and Tubulin in DEH-treated colorectal carcinoma cells with time gradient. And the densitometry of Western blotting bands of panel. All data were analyzed by using Unpaired Student's ttest and $p$-values less than 0.05 were considered as statistically significant. ${ }^{*} \mathrm{p}<0.05$, $* * \mathrm{p}<0.01, * * * \mathrm{p}<0.001$. 
Figure 3. DEH induces autophagy in colorectal cancer cells. A. Immunofluorescence staining of LC3B (green) and tubulin (red) in HCT 116 and SW620 cells treated with or without $60 \mu \mathrm{M}$ DEH for $48 \mathrm{~h}$. Nuclei were counterstained with DAPI (blue). Scale bars $=15 \mu \mathrm{m}$. Histogram shows quantification of the percentage of cells with LC3B puncta. B. Fluorescence images of GFP-MAP1LC3B puncta in HCT 116 and SW620 cells incubated with or without $60 \mu \mathrm{M}$ DEH for $48 \mathrm{~h}$. GFP-MAP1LC3B puncta were quantified and shown in the bar chart on the right. Scale bars $=10 \mu \mathrm{m}$. C. Autophagic vesicles detected by transmission electron microscope in HCT 116 and SW620 cells treated with or without $60 \mu \mathrm{M}$ DEH for $48 \mathrm{~h}$. Scale bar: $1 \mu \mathrm{m}$. N, nucleus. D. Protein levels of LC3B, p62, and ATG7 were detected by western blotting after HCT 116 and SW620 were treated with the indicated concentration of DEH for $48 \mathrm{~h}$. And the densitometry of western blotting is shown in the right of the pane. The statistical results are shown as means $\pm \mathrm{SD}$. All data were analyzed by using Unpaired Student's t-test and $\mathrm{p}$-values less than 0.05 were considered as statistically significant. ${ }^{*} \mathrm{p}<0.05$, $* * \mathrm{p}<0.01, * * * \mathrm{p}<0.001$.

Figure 4. DEH inhibits autophagic flux in colorectal cancer cells. A. Fluorescence imaging photographs of HCT 116 and SW620 cells infected with mRFP-GFP-LC3B recombinant adenovirus. Cells were infected with adenovirus for $24 \mathrm{~h}$, and then incubated with DMSO, EBSS, $10 \mu \mathrm{M}$ CQ and $60 \mu \mathrm{M}$ DEH respectively for $48 \mathrm{~h}$. Nuclei were stained with Hoechst 33342. Scale bar: $10 \mu \mathrm{m}$. And the average number of autophagosomes (yellow) and autolysosomes (red) per detected cell was counted. All data were analyzed by using Unpaired Student's t-test and p-values less than 0.05 were considered as statistically significant. ${ }^{*} \mathrm{p}<0.05,{ }^{* *} \mathrm{p}<0.01,{ }^{* * *} \mathrm{p}<0.001$. B. Western blot analysis of LC3B-II levels in HCT 116 and SW620 cells incubated with or without 60 $\mu \mathrm{M}$ DEH in the absence or presence of $10 \mathrm{mM} 3-\mathrm{MA}$ for $48 \mathrm{~h}$. And the western blot analysis of LC3B-II levels in HCT 116 and SW620 cells incubated with or without 60 $\mu \mathrm{M}$ DEH in the absence or presence of $10 \mu \mathrm{M}$ CQ for $48 \mathrm{~h}$. C. Western blot analysis of LC3B-II levels in HCT 116 and SW620 cells incubated in normal medium or EBSS with or without $60 \mu \mathrm{M}$ DEH for $6 \mathrm{~h}$. And the western blot analysis of LC3B-II levels in HCT 116 and SW620 cells incubated with or without $60 \mu \mathrm{M} \mathrm{DEH}$ in the absence or presence of $10 \mathrm{nM}$ BafA1 for $48 \mathrm{~h}$.

Figure 5. DEH induces ER stress in colorectal cancer cells. A. Gene set enrichment analysis of the UPR genes between control and DEH-treated cells. B. The thermodynamic chart of the mRNA expression level of genes related to ER stress in 
colorectal carcinoma cells after incubating with DEH for $48 \mathrm{~h}$. C. The subcellular structure of colorectal carcinoma cells after treatment with or without $60 \mu \mathrm{M} \mathrm{DEH}$ for $48 \mathrm{~h}$ were observed by electron microscopy. Scale bar: $2 \mu \mathrm{m}$. N, nucleus. The ER are labeled as circled in red. D. Western blotting assays were performed to detect the expression of BiP, Ero1-L $\alpha$, PERK, eIF2 $\alpha$, p-eIF2 $\alpha$, IRE1 $\alpha$, XBP-1s, CHOP, and Tubulin in HCT 116 and SW620 cells after treatment with or without DEH. And the densitometry of western blotting in the right panel. The data was shown as the means \pm SD. All data were analyzed by Unpaired Student's t-test and p-values less than 0.05 were considered as statistically significant. ${ }^{*} p<0.05,{ }^{* *} p<0.01,{ }^{* * *} p<0.001$.

Figure 6. EDH induces autophagy through PERK/eIF2 $\alpha$ and IRE1 $\alpha /$ XBP1s/CHOP pathways in colorectal cancer cells. A. MTT assay was used to evaluate the inhibition rate of colorectal carcinoma cells, which were transfected with PERK or IRE1 $\alpha$ siRNAs, followed by incubated with indicated concentrations of DEH for another $48 \mathrm{~h}$. B. The cell activity was detected by colony formation assay. Cells were transfected with PERK or IRE1 $\alpha$ siRNAs, followed by incubated with $60 \mu \mathrm{M}$ DEH for 10 days. Cells were stained with crystal violet staining solution. Scale bar: $200 \mathrm{~nm}$. The numbers of colonies were quantitated and shown in the right of the panel. C. Western blotting assay was used to detect the expression of IRE1 $\alpha$, LC3B, and Tubulin. Tubulin was used an internal control. D. Cells activity was detected by colony formation assay. Cells were pretreated with $4 \mathrm{U} 8 \mathrm{C}$, followed by incubated with DEH for 10 days. Cells were stained with crystal violet staining solution. Scale bar: $200 \mathrm{~nm}$. The numbers of colonies were quantitated and shown in the below of the panel. E. The expression of IRE1 $\alpha$, LC3B- II , and Tubulin were detected after DEH treatment with the inhibitor of IRE1 $\alpha, 4$ U8C or DMSO for $48 \mathrm{~h}$. All data were analyzed by using Unpaired Student's t-test and p-values less than 0.05 were considered as statistically significant. ${ }^{*} \mathrm{p}<0.05$, $* * \mathrm{p}<0.01, * * * \mathrm{p}<0.001$.

Figure 7. Effects of DEH in the growth of colorectal cancer in vivo. A. HCT 116, SW620, and one tumor tissue from colon cancer patient were injected or transplanted into the frank of NOD/SCID mice respectively. The tumor-bearing mice were treated with DMSO or $40 \mathrm{mg} / \mathrm{kg}$ DEH by intraperitoneal injection when tumors were palpable. Tumor volume was measured every three days. Two weeks later, the mice were anesthetized and killed, tumors were imaged and analyzed. B. Hematoxylin and eosin (H\&E) staining of the indicated xenograft tumors. Scale bar: $50 \mu \mathrm{m}$. C. Immunohistochemical (IHC) staining of the indicated xenograft tumors. Scale bar: 50 
$\mu \mathrm{m}$. D. H\&E staining of the heart, liver, spleen, lung, and kidney in mice treated with DMSO or $50 \mathrm{mg} / \mathrm{kg}$ DEH. Scale bar: $50 \mu \mathrm{m}$. E. The expression of BiP, PERK and IRE1 $\alpha$ of xenograft tumor was detected by western blotting. F. The expression of LC3B, p62 and Tubulin in xenograft tumor was detected by western blotting. All data are means \pm S.D. and are representative of 3 independent experiments. $P$ value $<0.05$ was considered significant. $* * \mathrm{P}<0.01 ; * * * \mathrm{P}<0.001$.

Figure 8. The mechanism prediction diagram of DEH treatment in colorectal cancer.

Supplementary materials: The following contents are supplementary materials information. Figure S1. DEH inhibits cell growth of not through apoptosis in colorectal cancer cells. A. The chemical structure of DEH used in this study. B. The plate colony formation assay was used to evaluate the cell activity after treatment with DMSO or DEH. The numbers of colonies were quantitated and shown in the right of the panel. C. HCT 116 and SW620 cells were incubated with DMSO or DEH for 2 days, and the cell apoptosis was demonstrated by Annexin V/PI staining with flow cytometry. The cell apoptosis statistics was listed in the right of the panel. All data are means \pm S.D. and are representative of 3 independent experiments. $\mathrm{P}$ value $<0.05$ was considered significant. ns, there was no significant difference, ${ }^{* * *} \mathrm{P}<0.001$. Figure S2. Expression profiles of autophagic gene and associated gene of colorectal cancer cells after treatment with DMSO or DEH. A. A heatmap of autophagic gene and associated genes of HCT 116 and SW620 cells after incubated with DMSO or DEH for 2 days. B. Western blotting were performed to investigate the expression of LC3B, p62, ATG7, and Tubulin in HCT 116 and SW620 cells after treatment with DEH. C. Western blotting were performed to investigate the expression of BiP, PERK, IRE1 $\alpha$, and Tubulin in HCT 116 and SW620 cells after treatment with DEH. Tubulin was used as a control. Figure S3. The anticancer activity of DEH on colorectal cancer were evaluated by using CDX and PDX model in vivo. A. Quantified results of tumor weight. B. The Ki67 positive cells of tumor-bearing mice was treatment with DMSO or DEH were quantified and shown in the bar chart. C. The weight of tumor-bearing mice was measured after treatment with DMSO or DEH for 2 weeks. All data are means \pm S.D. and are representative of 3 independent experiments. $\mathrm{P}$ value $<0.05$ was considered significant. $* * \mathrm{P}<0.01 ; * * * \mathrm{P}<0.001$.

Authors' contributions: CL performed experiments, wrote the manuscript and 
contributed to the design of the study. KZ designed the study, performed animal experiments and revised this manuscript. GP performed plasmid construction, lentivirus packaging, concentration and infection. HJ, CL and XW performed cell culture. YW, RL and LD critically reviewed the manuscript. LY and HC supervised the study and revised the manuscript. All authors read and approved the final manuscript.

Funding: This work was supported by the National Key Research and Development Program of China (2016YFC1302204, 2017YFC1308601), the National Natural Science Foundation of China (31672496, 31802142, 81872071, and 81672502), project funded by the Fundamental Research Funds for the Central Universities (XDJK2019C089), the China Postdoctoral Science Foundation (2019T120801 and 2017M620408), Chongqing Natural Science foundation of Chongqing (cstc2019jcyjzdxmX0033) and Chongqing University Innovation Team Building Program funded projects (CXTDX201601010).

Acknowledgements: We thank The Ninth People's Hospital of Chongqing for providing patient tumor samples. We are also very grateful to Erhu Zhao, Xiong Weng and Xin Hu for technical support and helpful comments.

Conflicts of interests: The authors declare that they have no competing interests

Abbreviations: DEH, Dehydrodiisoeugenol; CRC, Colorectal cancer; ER, Endoplasmic reticulum; ERS, Endoplasmic reticulum stress; PBS, phosphate buffer solution.

\section{References}

1. Gao, C.; Cao, W.; Bao, L.; Zuo, W.; Xie, G.; Cai, T.; Fu, W.; Zhang, J.; Wu, W.; Zhang, X.J.N.c.b. Autophagy negatively regulates Wnt signalling by promoting Dishevelled degradation. 2010, 12, 781-790.

2. Levy, J.M.M.; Towers, C.G.; Thorburn, A.J.N.R.C. Targeting autophagy in cancer. 2017, 17, 528542.

3. Rogov, V.; Dötsch, V.; Johansen, T.; Kirkin, V.J.M.c. Interactions between autophagy receptors and ubiquitin-like proteins form the molecular basis for selective autophagy. 2014, 53, 167178.

4. Kim, I.; Xu, W.; Reed, J.C.J.N.r.D.d. Cell death and endoplasmic reticulum stress: disease relevance and therapeutic opportunities. 2008, 7, 1013-1030.

5. Shimodaira, Y.; Takahashi, S.; Kinouchi, Y.; Endo, K.; Shiga, H.; Kakuta, Y.; Kuroha, M.; Shimosegawa, T.J.B.; communications, b.r. Modulation of endoplasmic reticulum (ER) stressinduced autophagy by C/EBP homologous protein (CHOP) and inositol-requiring enzyme $1 \alpha$ (IRE1 $\alpha$ ) in human colon cancer cells. 2014, 445, 524-533.

6. Li, T.; Su, L.; Zhong, N.; Hao, X.; Zhong, D.; Singhal, S.; Liu, X.J.A. Salinomycin induces cell 
death with autophagy through activation of endoplasmic reticulum stress in human cancer cells. 2013, 9, 1057-1068.

7. Høyer-Hansen, M.; Jäättelä, M.J.C.D.; Differentiation. Connecting endoplasmic reticulum stress to autophagy by unfolded protein response and calcium. 2007, 14, 1576-1582.

8. Krishnamachary, B.; Berg-Dixon, S.; Kelly, B.; Agani, F.; Feldser, D.; Ferreira, G.; lyer, N.; LaRusch, J.; Pak, B.; Taghavi, P.J.C.r. Regulation of colon carcinoma cell invasion by hypoxiainducible factor 1. 2003, 63, 1138-1143.

9. Smeby, J.; Sveen, A.; Merok, M.A.; Danielsen, S.A.; Eilertsen, I.A.; Guren, M.; Dienstmann, R.; Nesbakken, A.; Lothe, R.A.J.A.o.O. CMS-dependent prognostic impact of KRAS and BRAF V600E mutations in primary colorectal cancer. 2018, 29, 1227-1234.

10. Chen, X.-z.; Cao, Z.-y.; Liao, L.-m.; Liu, Z.-z.; Du, J.J.C.j.o.i.m. Application of serum pharmacology in evaluating the antitumor effect of Fuzheng Yiliu Decoction (扶正抑瘤方) from Chinese medicine. 2014, 20, 450-455.

11. Wang, S.-Y.; Shiao, M.-S.J.J.o.F.; Analysis, D. Pharmacological functions of Chinese medicinal fungus Cordyceps sinensis and related species. 2000, 8.

12. Li, F.; Yang, X.W.J.B.C. Analysis of anti - inflammatory dehydrodiisoeugenol and metabolites excreted in rat feces and urine using HPLC - UV. 2012, 26, 703-707.

13. Murakami, Y.; Shoji, M.; Hirata, A.; Tanaka, S.; Yokoe, I.; Fujisawa, S.J.A.o.b.; biophysics. Dehydrodiisoeugenol, an isoeugenol dimer, inhibits lipopolysaccharide-stimulated nuclear factor kappa B activation and cyclooxygenase-2 expression in macrophages. 2005, 434, 326332.

14. Yang, A.-H.; He, X.; Chen, J.-X.; He, L.-N.; Jin, C.-H.; Wang, L.-L.; Zhang, F.-L.; An, L.-J.J.C.-b.i. Identification and characterization of reactive metabolites in myristicin-mediated mechanism-based inhibition of CYP1A2. 2015, 237, 133-140.

15. Patel, H.; Gupte, A. Laccase Catalysis: A Green Approach in Bioactive Compound Synthesis. In Biotechnology: Concepts, Methodologies, Tools, and Applications, IGI Global: 2019; pp. 20542089.

16. Fang, Z.; Gong, C.; Yu, S.; Zhou, W.; Hassan, W.; Li, H.; Wang, X.; Hu, Y.; Gu, K.; Chen, X.J.C.I. NFYB-induced high expression of E2F1 contributes to oxaliplatin resistance in colorectal cancer via the enhancement of CHK1 signaling. 2018, 415, 58-72.

17. Choi, J.H.; Won, Y.W.; Kim, H.S.; Oh, Y.H.; Lim, S.; Kim, H.J.J.O.I. Oxaliplatin-induced sinusoidal obstruction syndrome mimicking metastatic colon cancer in the liver. 2016, 11, 2861-2864.

18. Al-Rawi, S.S.; Ibrahim, A.H.; Ab Rahman, N.N.N.; Nama, M.M.B.; Majid, A.M.A.; Ab Kadir, M.O.J.P.F.S. The effect of supercritical fluid extraction parameters on the nutmeg oil extraction and its cytotoxic and antiangiogenic properties. 2011, 1, 1946-1952.

19. Simmonds, P.; Best, L.; George, S.; Baughan, C.; Buchanan, R.; Davis, C.; Fentiman, I.; Gosney, M.; Northover, J.; Williams, C.J.T.L. Surgery for colorectal cancer in elderly patients: a systematic review. 2000, 356, 968-974.

20. Li, F.; Yang, X.-W.; Krausz, K.W.; Nichols, R.G.; Xu, W.; Patterson, A.D.; Gonzalez, F.J.J.J.o.p.r. Modulation of colon cancer by nutmeg. 2015, 14, 1937-1946.

21. Schepetkin, I.A.; Kushnarenko, S.V.; Özek, G.; Kirpotina, L.N.; Sinharoy, P.; Utegenova, G.A.; Abidkulova, K.T.; Özek, T.; Başer, K.H.s.C.; Kovrizhina, A.R.J.J.o.a., et al. Modulation of human neutrophil responses by the essential oils from Ferula akitschkensis and their constituents. 2016, 64, 7156-7170. 
22. Sepulveda, A.R.; Hamilton, S.R.; Allegra, C.J.; Grody, W.; Cushman-Vokoun, A.M.; Funkhouser, W.K.; Kopetz, S.E.; Lieu, C.; Lindor, N.M.; Minsky, B.D.J.A.j.o.c.p. Molecular biomarkers for the evaluation of colorectal cancer: guideline from the American Society for Clinical Pathology, College of American Pathologists, Association for Molecular Pathology, and American Society of Clinical Oncology. 2017, 147, 221-260.

23. El-Alfy, A.T.; Wilson, L.; EISohly, M.A.; Abourashed, E.A.J.J.o.e. Towards a better understanding of the psychopharmacology of nutmeg: activities in the mouse tetrad assay. 2009, 126, 280286.

24. Mikhaylova, O.; Stratton, Y.; Hall, D.; Kellner, E.; Ehmer, B.; Drew, A.F.; Gallo, C.A.; Plas, D.R.; Biesiada, J.; Meller, J.J.C.c. VHL-regulated MiR-204 suppresses tumor growth through inhibition of LC3B-mediated autophagy in renal clear cell carcinoma. 2012, 21, 532-546.

25. Prakash, E.; Gupta, D.K.J.J.o.F.; Science, N. Cytotoxic activity of ethanolic extract of Myristica fragrans (Houtt) against seven human cancer cell lines. 2013, 1, 1-3.

26. Wang, F.; Jiang, J.; Hu, S.; Ma, H.; Zhu, H.; Tong, Q.; Cheng, L.; Hao, X.; Zhang, G.; Zhang, Y.J.F. Secondary metabolites from endophytic fungus Chaetomium sp. induce colon cancer cell apoptotic death. 2017, 121, 86-93.

27. Yin, C.; Ke, X.; Zhang, R.; Hou, J.; Dong, Z.; Wang, F.; Zhang, K.; Zhong, X.; Yang, L.; Cui, H.J.T.F.J. G9a promotes cell proliferation and suppresses autophagy in gastric cancer by directly activating mTOR. 2019, 33, 14036-14050.

28. Li, C.; Deng, C.; Pan, G.; Wang, X.; Zhang, K.; Dong, Z.; Zhao, G.; Tan, M.; Hu, X.; Shi, S.J.J.o.E., et al. Lycorine hydrochloride inhibits cell proliferation and induces apoptosis through promoting FBXW7-MCL1 axis in gastric cancer. 2020, 39, 1-16.

29. Yang, J.; Dong, Z.; Ren, A.; Fu, G.; Zhang, K.; Li, C.; Wang, X.; Cui, H.J.J.o.C.; Medicine, M. Antibiotic tigecycline inhibits cell proliferation, migration and invasion via down - regulating CCNE2 in pancreatic ductal adenocarcinoma. 2020, 24, 4245-4260.

30. Winawer, S.J.; Fletcher, R.H.; Miller, L.; Godlee, F.; Stolar, M.; Mulrow, C.; Woolf, S.; Glick, S.; Ganiats, T.; Bond, J.J.G. Colorectal cancer screening: clinical guidelines and rationale. 1997, 112, 594-642.

31. Moscat, J.; Diaz-Meco, M.T.J.C. p62 at the crossroads of autophagy, apoptosis, and cancer. 2009, 137, 1001-1004.

32. Zhang, K.; Hu, X.; Zhao, Y.; Pan, G.; Li, C.; Ji, H.; Li, C.; Yang, L.; Abbas, M.N.; Cui, H.J.D., et al. Scavenger receptor $\mathrm{B} 8$ improves survivability by mediating innate immunity in silkworm, Bombyx mori. 2020, 103917.

33. Lv, Q.-Q.; Yang, X.-N.; Yan, D.-M.; Liang, W.-Q.; Liu, H.-N.; Yang, X.-W.; Li, F.J.J.o.P.; Analysis, B. Metabolic profiling of dehydrodiisoeugenol using xenobiotic metabolomics. 2017, 145, 725733.

34. Codogno, P.; Meijer, A.J.J.C.m. Autophagy: a potential link between obesity and insulin resistance. 2010, 11, 449-451.

35. Rabinowitz, J.D.; White, E.J.S. Autophagy and metabolism. 2010, 330, 1344-1348.

36. Levine, B.; Kroemer, G.J.C. Autophagy in the pathogenesis of disease. 2008, 132, 27-42.

37. Panda, P.K.; Mukhopadhyay, S.; Das, D.N.; Sinha, N.; Naik, P.P.; Bhutia, S.K. Mechanism of autophagic regulation in carcinogenesis and cancer therapeutics. In Proceedings of Seminars in cell \& developmental biology; pp. 43-55.

38. Maheswari, U.; Ghosh, K.; Sadras, S.R.J.A. Licarin A induces cell death by activation of 
autophagy and apoptosis in non-small cell lung cancer cells. 2018, 23, 210-225.

39. Pettersen, K.; Andersen, S.; van der Veen, A.; Nonstad, U.; Hatakeyama, S.; Lambert, C.; Lach - Trifilieff, E.; Moestue, S.; Kim, J.; Grønberg, B.H.J.J.o.c., sarcopenia, et al. Autocrine activin A signalling in ovarian cancer cells regulates secretion of interleukin 6 , autophagy, and cachexia. 2020, 11, 195-207.

40. New, M.; Tooze, S.J.B. The Role of Autophagy in Pancreatic Cancer-Recent Advances. 2020, $9,7$.

41. von Haehling, S.; Morley, J.E.; Coats, A.J.; Anker, S.D.J.J.o.c., sarcopenia; muscle. Ethical guidelines for publishing in the Journal of Cachexia, Sarcopenia and Muscle: update 2017. 2017, 8, 1081-1083.

42. Pal, M.; Febbraio, M.A.; Whitham, M.J.I.; biology, c. From cytokine to myokine: the emerging role of interleukin - 6 in metabolic regulation. 2014, 92, 331-339.

43. Lach-Trifilieff, E.; Minetti, G.C.; Sheppard, K.; Ibebunjo, C.; Feige, J.N.; Hartmann, S.; Brachat, S.; Rivet, H.; Koelbing, C.; Morvan, F.J.M., et al. An antibody blocking activin type II receptors induces strong skeletal muscle hypertrophy and protects from atrophy. 2014, 34, 606-618.

44. Shintani, T.; Klionsky, D.J.J.S. Autophagy in health and disease: a double-edged sword. 2004, 306, 990-995.

45. Zhang, K.; Fu, G.; Pan, G.; Li, C.; Shen, L.; Hu, R.; Zhu, S.; Chen, Y.; Cui, H.J.C.d.; disease. Demethylzeylasteral inhibits glioma growth by regulating the miR-30e-5p/MYBL2 axis. 2018, 9, 1-14.

46. Daniels, M.S.; Bannon, S.A.; Mork, M.E.J.J.o.C.O. Frequency of germline BRCA1/2 mutations in unselected patients with colorectal cancer. 2017, 35, 2588-2588.

47. Towers, C.G.; Wodetzki, D.; Thorburn, A.J.T.J.o.c.b. Autophagy and cancer: Modulation of cell death pathways and cancer cell adaptations Autophagy and cancer. 2020, 219.

48. Ávalos, Y.; Canales, J.; Bravo-Sagua, R.; Criollo, A.; Lavandero, S.; Quest, A.F.J.B.r.i. Tumor suppression and promotion by autophagy. 2014, 2014.

49. Yang, Y.; Klionsky, D.J.J.C.D.; Differentiation. Autophagy and disease: Unanswered questions. 2020, 1-14. 